\title{
Drug-drug Interactions in Kidney Transplant Patients with Coronavirus Disease 2019: A Report of Two Cases and Literature Review
}

\author{
Shahriyar Shahbazi Khamas ${ }^{1}$, Elham Ramezanzadeh ${ }^{2}$ and Atefeh Jafari ${ }^{3, *}$ \\ ${ }^{1}$ Student Research Committee, School of Pharmacy, Guilan University of Medical Sciences, Rasht, Guilan, Iran \\ 2 Razi Clinical Research Development Center, Guilan University of Medical Sciences, Rasht, Iran \\ ${ }^{3}$ Department of Clinical Pharmacy, School of Pharmacy, Guilan University of Medical Sciences, Rasht, Iran
}

* Corresponding author: Atefeh Jafari, Department of Clinical Pharmacy, School of Pharmacy, Guilan University of Medical Sciences, Rasht, Iran. Tel: 00981333486470; Email: atf.jafari@gmail.com

Received 2021 January 14; Revised 2021 February 01; Accepted 2021 February 29.

\begin{abstract}
Introduction: On February 19, 2020, the first confirmed case of Coronavirus disease 2019, known as COVID-19, was identified in Iran. The disease spread rapidly throughout the country. Some of the cases were asymptomatic, some had mild to severe symptoms, and some of them died. Transplant patients are highly at risk due to long-term immunosuppressive therapy, and precise treatment approaches are needed to not only cure the disease but also protect graft function.

Case Presentation: This study reports two kidney transplant patients with COVID-19 pneumonia, both of whom showed respiratory and gastrointestinal symptoms. High cyclosporine and tacrolimus trough levels were observed despite initial dose reduction. After a treatment program containing reduced immunosuppressant dose and the addition of pulsatile hydrocortisone, these patients recovered effectively. We also discuss the importance of drug-drug interactions related to COVID-19 treatment protocol medications, especially with immunosuppressants in these patients.

Conclusion: In conclusion, frequent monitoring of the trough levels of calcineurin and mammalian target of rapamycin inhibitors during hospitalization is recommended. It helps to determine the ideal treatment and prevent serious clinical toxicity.

Keywords: COVID-19, Drug interaction, Kidney transplantation, Pneumonia
\end{abstract}

\section{Introduction}

As of March 11, 2020, coronavirus disease 2019 (COVID-19) was declared a global pandemic; subsequently, countries have been carrying the heavy burden of the COVID-19 outbreak. Kidney transplant patients are at high risk due to their immunosuppressive regimens. Treatment and prognosis of COVID-19 in transplant patients may differ from others due to long-term transplantrelated immunosuppression (1). For all we know, the first report of COVID-19 in kidney transplant recipients was a 52-year-old male from Wuhan with similar clinical characteristics to those of nontransplant COVID-19 patients; he improved 13 days after hospital admission (2).

Previous studies recommended that immunosuppression might weaken the "immune system overdrive", underlie the development of acute respiratory distress syndrome, and lead to mortality (3). These patients receive many medications either for immunosuppression or for the treatment of the underlying disease; therefore, they are usually struggling with polypharmacy and drug interactions. Calcineurin inhibitors (CNIs) have the most potential to be affected by drug-drug interactions (DDIs) and have a narrow therapeutic window (4).

The COVID-19 treatment protocols include antiviral drugs causing many DDIs, especially pharmacokinetics in nature. Inhibition of the cytochrome P450 enzyme system leading to a surge in serum concentrations of CNIs may increase the risk of side effects, including neurotoxicity and nephrotoxicity (5). Examination of COVID-19 patients on antivirals can help us study the effect of lopinovir/ritonivir, which was previously mainly used in AIDS patients, in a wider aspect. Moreover, it helps us study the effects of drug interaction on the therapeutic effects and changes in the levels of CNIs and mammalian target of rapamycin (m-TOR) inhibitors. As the literature on kidney transplant cases infected with COVID-19 is limited, we believe that sharing the experiences of these patients from Iran may be of interest to healthcare providers all over the world.

\section{Case Presentation}

\subsection{Case 1}

M was a 53-year-old man who underwent kidney transplantation 15 years ago. Triple maintenance immunosuppressive therapy was administrated to him with oral cyclosporine (75 mg twice daily), mycophenolate mofetil (1000 mg twice daily), and prednisolone ( $5 \mathrm{mg}$ daily) until last year. From a year ago he ceased using prednisolone and did not visit a physician again. In his last visit, his serum creatinine (Cr) was $2.1 \mathrm{mg} / \mathrm{dL}$ and cyclosporine levels were in therapeutic ranges.

On March 17, 2020, the patient was transferred from another hospital to Razi Educational Remedial 
and Research Center, a governmental referral teaching hospital affiliated with Guilan University of Medical Sciences, Rasht, Iran. He had been taking oseltamivir (75 mg every other day) and lopinavir/ritonavir (400 $\mathrm{mg} / 100 \mathrm{mg}$ twice daily) from seven and four days before his admission, respectively. At the time of admission, his immunosuppressive therapy was adjusted, cyclosporine was changed to $25 \mathrm{mg}$ twice daily, and mycophenolate mofetil was discontinued.

His initial symptoms, recorded in a monitoring sheet, were shortness of breath, dry coughs, fever, diarrhea, and agitation. Physical examination on admission revealed a body temperature of $36.9{ }^{\circ} \mathrm{C}$ and oxygen saturation of $92 \%$ with the oxygen mask. His laboratory results and radiological features on admission were as follows: white blood cell (WBC) count on peripheral blood: $5.6 \times 10^{9} / \mathrm{L}$ (lymphocyte: $10 \%$ ), Cr level: $4.2 \mathrm{mg} / \mathrm{dL}$; C-reactive protein (CRP): 2+, LDH: $1625 \mathrm{U} / \mathrm{L}$, alanine aminotransferase (ALT): $47 \mathrm{U} / \mathrm{L}$, and aspartate aminotransferase (AST): 61 U/L. Chest computed tomography (CT) showed ground-glass opacity with multifocal and bilateral consolidation, linear opacity bronchiectasis, and a CT score of 20. Nasopharyngeal swab sample for reverse real-time polymerase chain reaction (PCR) for SARSCoV-2 was negative. However, based on laboratory and clinical characteristics, the patient was diagnosed with COVID-19 pneumonia.

The patient received treatment based on the national protocol at that time which included lopinavir/ritonavir (400 mg/100 mg twice daily) along with hydroxychloroquine (400 mg stat). In addition, meropenem $500 \mathrm{mg}$ twice daily and vancomycin 1000 mg stat, $500 \mathrm{mg}$ every $48 \mathrm{~h}$ were administrated. Moreover, according to recommendations (6), cyclosporine was reduced to $50 \mathrm{mg}$ twice a day, mycophenolate mofetil was ceased, and hydrocortisone (50mg thrice daily) was also initiated (Figure 1).

On day four, dry coughs worsened, and sodium and potassium levels dropped to $131 \mathrm{mEq} / \mathrm{L}$ and 3.2 $\mathrm{mEq} / \mathrm{L}$, respectively. Hence, dextromethorphan 15 mg thrice daily and vancomycin $500 \mathrm{mg}$ were commenced. The day after, metronidazole $500 \mathrm{mg}$ thrice daily was started due to diarrhea. On day seven, intravenous immunoglobulin was added to his drug regimen. Proceeding to day 10 , the cyclosporine level $\left(\mathrm{C}_{0}\right)$ was $414.1 \mathrm{ng} / \mathrm{dL}$; therefore, the cyclosporine dose was reduced to $25 \mathrm{mg}$ twice a day and corticosteroid therapy was changed to prednisolone (5 mg twice daily).

In the following days, he showed general improvement with the following results: oxygen saturation: $97 \%$ (room air), $\mathrm{Cr}: 2.3 \mathrm{mg} / \mathrm{dL}, \mathrm{C}_{0}: 29.9$ ng/dL, ALT: 22 U/L, and AST: 25 U/L. On day 27, immunosuppressive therapy was restarted and three days later, he was discharged from the hospital. His immunosuppressive regimen included prednisolone (5 mg twice daily), cyclosporine (50 mg twice daily), and mycophenolate mofetil (1000 mg twice daily).

A week later, in his follow-up in the office of the physician, he was in good condition without any respiratory symptoms. His $\mathrm{C}_{0}$ was $64.9 \mathrm{ng} / \mathrm{dL}$ and the other laboratory findings were as follows: $\mathrm{Cr}$ : 3.2 $\mathrm{mg} / \mathrm{dL}$, blood urea nitrogen: $54 \mathrm{mg} / \mathrm{dL}$, and WBC count: $11 \times 10^{9} /$ L (lymphocyte: $26 \%$ ).

\subsection{Case 2}

A was a 44-year-old man who underwent kidney transplantation in 2011 with a baseline $\mathrm{Cr}$ of 1.3 $\mathrm{mg} / \mathrm{dL}$ and was admitted on March 14, 2020. His initial symptoms were dry coughs, fever, and diarrhea. Physical examination on admission revealed a body temperature of $37{ }^{\circ} \mathrm{C}$ and oxygen saturation of $96 \%$ (ambient air). His chest CT results showed multifocal and bilateral consolidation, bronchiectasis, and a CT score of 16.

The baseline regimen included sirolimus (1 mg twice daily), prednisolone (5 mg daily), and mycophenolate sodium (720 mg twice daily). His treatment included lopinavir/ritonavir (400 mg/100 mg twice daily), along with hydroxychloroquine (400 $\mathrm{mg}$ stat) and oseltamivir (75mg twice daily). For adjustments to immunosuppressive therapy, sirolimus was reduced to $1 \mathrm{mg}$ daily, mycophenolate sodium was discontinued, and hydrocortisone (50 mg thrice daily) was also initiated (Figure 2). Nasopharyngeal swab for PCR for SARS-CoV-2 was negative based on laboratory and clinical characteristics. He was discharged with the diagnosis of COVID-19 and home isolation was prescribed for him. Moreover, he continued hydroxychloroquine and oseltamivir, discontinued lopinavir/ritonavir, and started immunosuppressive therapy with pre-illness dosage the day after.

After three days, the patient returned with

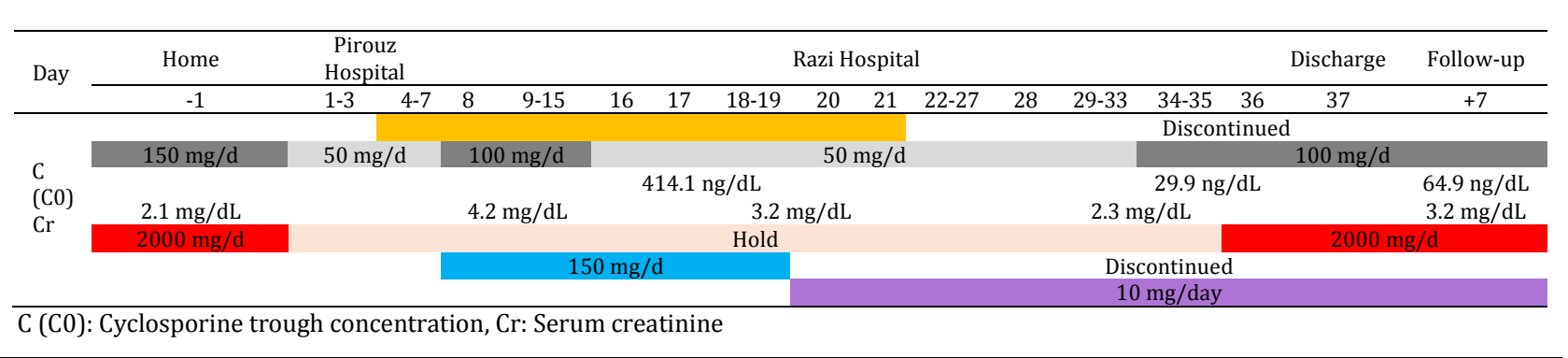

Figure 1. Treatment and immunosuppressive dose adjustment for (M) according to the day of illness 


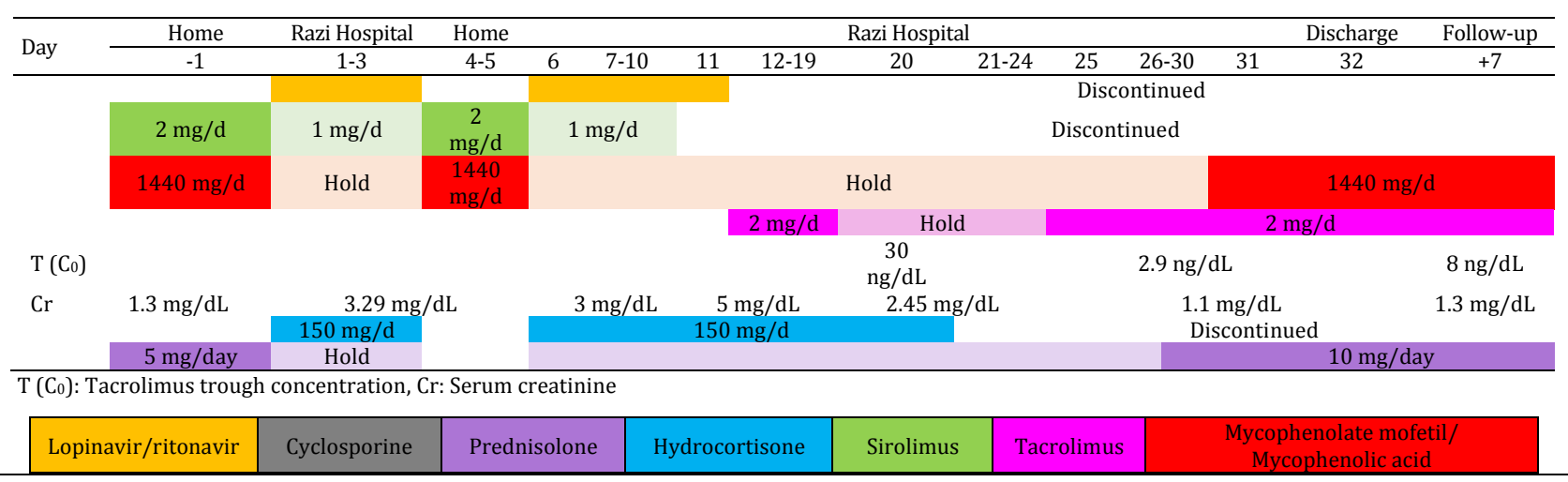

Figure 2. Treatment and immunosuppressive dose adjustment for $(\mathrm{A})$ according to the day of illness

shortness of breath, dry coughs, myalgia, and severe diarrhea. The blood workup were as follows: WBC count: $8.8 \times 10^{9} / \mathrm{L}$ (lymphocyte: $13 \%$ ), Cr level: 3 mg/dL, CRP: 3+, alkaline phosphatase: 133 IU/L, ALT: $75 \mathrm{U} / \mathrm{L}$, and AST: $87 \mathrm{U} / \mathrm{L}$. Hydroxychloroquine (400 $\mathrm{mg}$ stat $)$, lopinavir/ritonavir $(400 \mathrm{mg} / 100 \mathrm{mg}$ twice daily), and meropenem $1000 \mathrm{mg}$ twice daily were started. On day 19, based on recommendations and due to m-TOR inhibitors potentials for leukopenia, thrombocytopenia, and pneumonitis (7), sirolimus was replaced by tacrolimus ( $1 \mathrm{mg}$ twice daily).

In the following days, shortness of breath worsened and oxygen saturation dropped to $88 \%$ in ambient air; therefore, metronidazole $(500 \mathrm{mg}$ thrice daily) and levofloxacin (500 mg daily) were started. On day 20, tacrolimus level was above $30 \mathrm{ng} / \mathrm{dL}$; hence, tacrolimus usage was ceased and restarted again after six days. On day 25 , the patient presented a general improvement, including a significant decrease in cough and shortness of breath with an oxygen saturation level of $94 \%$ (room air), $\mathrm{Cr}$ of 1.1 $\mathrm{mg} / \mathrm{dL}$, tacrolimus level of $2.9 \mathrm{ng} / \mathrm{dL}, \mathrm{ALT}$ of $17 \mathrm{U} / \mathrm{L}$, and AST of $39 \mathrm{U} / \mathrm{L}$. A week later, He was discharged and tacrolimus (1 mg twice daily), mycophenolate sodium (360 mg twice daily), and prednisolone (5 mg twice daily) were prescribed for him. In the followup, tacrolimus level was $8 \mathrm{ng} / \mathrm{dL}$, and he was in good health at home.

\section{Discussion}

As a result of immunosuppression in kidney transplant patients, the clinical manifestations, treatment, and prognosis of COVID-19 pneumonia in them may vary from other patients. Nevertheless, the available data on COVID-19 in these patients is not sufficient.

In a case report, Meziyerh $\mathrm{S}$ et al. reported a 35-year-old kidney transplant man who had severe COVID-19 pneumonia with initial symptoms of fever, cough, malaise, muscle pain, and headache. His maintenance therapy included everolimus $(3 \mathrm{mg}$ twice daily) and prednisolone (7.5 mg daily). His baseline $\mathrm{Cr}$ level was around $1.8 \mathrm{mg} / \mathrm{dL}$, and everolimus trough concentrations were within the range of 3-8 $\mu \mathrm{g} / \mathrm{L}$. Symptoms worsened over time; accordingly, $\mathrm{Cr}$ raised to $3.6 \mathrm{mg} / \mathrm{dL}$, and a nasopharyngeal swab for SARS-CoV-2 was positive. Therefore, antiviral therapy with chloroquine (600 mg stat and $300 \mathrm{mg}$ twice daily afterward) and lopinavir/ritonavir (400/100 mg twice daily) was started and everolimus was reduced to $2 \mathrm{mg}$ twice daily.

After two days, the everolimus trough level was $31.1 \mu \mathrm{g} / \mathrm{L}$; hence, everolimus was discontinued. However, two days after the discontinuation, the everolimus trough level was $14.4 \mu \mathrm{g} / \mathrm{L}$. Therefore, lopinavir/ritonavir and chloroquine were discontinued as well. Four days later, the everolimus level was below the detection limit, and cyclosporine was started for immunosuppression (8). This team preferred cyclosporine over everolimus due to its potential in vitro antiviral effect against coronaviruses, patient clinical condition, and adverse effects of everolimus, like mucosal ulcers and interstitial lung disease.

In another study, Bartiromo $\mathrm{M}$ et al. reported a 36-year-old female kidney transplant patient diagnosed with COVID-19 pneumonia with initial symptoms of fatigue, dry cough, and coryza. In her last visit, her $\mathrm{Cr}$ and tacrolimus trough levels were $1.5 \mathrm{mg} / \mathrm{dL}$ and $8.8 \mathrm{ng} / \mathrm{mL}$, respectively, with maintenance therapy including tacrolimus $(5 \mathrm{mg}$ twice daily) and methylprednisolone (4 mg daily). On admission, her Cr level was $2.29 \mathrm{mg} / \mathrm{dL}$, and antiviral therapy was started with lopinavir/ritonavir $(400 \mathrm{mg} / 100 \mathrm{mg}$ twice daily) along with hydroxychloroquine (200 mg twice daily).

Adjustments were as follows: tacrolimus and methylprednisolone were reduced to $3 \mathrm{mg}$ twice daily and $6.5 \mathrm{mg}$ daily, respectively. Two days later, due to drug intolerance, darunavir/cobicistat $(800$ $\mathrm{mg} / 150 \mathrm{mg}$ ) were replaced by lopinavir/ritonavir. On day three, the tacrolimus trough level was 90.5 $\mathrm{ng} / \mathrm{mL}$; therefore, both antiviral therapy and tacrolimus were discontinued. Daily monitoring of tacrolimus trough levels showed a gradual reduction on days 4 to $9(81.5,74.5,72.4,33.8,18.8 \mathrm{ng} / \mathrm{mL}$ in 
that order). She was discharged on day nine, and tacrolimus trough levels on days 16, and 23 were 19, and $15 \mathrm{ng} / \mathrm{mL}$, respectively, while $\mathrm{Cr}$ on day 23 was $1.75 \mathrm{mg} / \mathrm{dL}$.

In the aforementioned study, the team focused on the reduction of immunosuppressant use and maintenance of the low dose of methylprednisolone. In this method, the anti-infection immunity was restored in patients, and the anti-inflammatory and graft-protective effects of steroids led to clinical improvement (9). They also found extremely high tacrolimus level (10-fold the baseline through level) even after reduction to $3 / 5$ background dose, which is probably due to the co-administration of lopinavir/ritonavir. In the present study, we also reduced the dosage of cyclosporine and sirolimus, withdrew mycophenolate sodium or mycophenolate mofetil, and used appropriate doses of pulsatile intravenous corticosteroids. It should be kept in mind that the usage of excessive doses of steroids for long durations may deteriorate recovery and lead to other side effects (10).

Protease inhibitors are known to inhibit the cytochrome 3A enzyme system that is responsible for the metabolism of immunosuppressive drugs, such as tacrolimus, sirolimus, and cyclosporine (11). In the first visit of $\mathrm{A}$, he was in good condition and was discharged to continue his treatment at home, and sirolimus was not changed to tacrolimus at that time. His exacerbated pneumonia and return to the hospital could confirm the m-TOR inhibitor potential for pneumonitis (12).

Given that $\mathrm{m}$-TOR inhibitors may increase mucosal susceptibility more precisely, as evidenced by adverse events, such as mucosal ulcers, leukopenia, thrombocytopenia, and pneumonitis, we decided to use tacrolimus instead of sirolimus. Two weeks after the discontinuation of lopinavir/ ritonavir, the tacrolimus level was about four-fold above the therapeutic ranges. Our result is inconsistent with that of a study performed by Jain $\mathrm{AB}$ et al., according to which the patients may not need a further tacrolimus dose for up to three weeks after the start of lopinavir/ritonavir (13). With our estimations according to recommendations, our patient may need 0.5-1 mg tacrolimus per week while he was on lopinavir/ritonavir (14).

When $\mathrm{M}$ was in the hospital and took cyclosporine (100 mg daily), lopinavir/ritonavir $(400 \mathrm{mg} / 100 \mathrm{mg}$ twice daily), and hydroxychloroquine ( $400 \mathrm{mg}$ ), his cyclosporine level was about six times higher than when he was discharged and took $100 \mathrm{mg}$ cyclosporine daily at home. The difference between the multiplication of cyclosporine concentration in this study and the one conducted by Vogel $\mathrm{M}$ et al. may be due to the co-administration of hydroxychloroquine (15). He took only $400 \mathrm{mg}$; however, its long half-life leads to interaction even weeks after withdrawal (16). With regard to recommendations, our patient might have needed cyclosporine (25 mg every two days) while he was on lopinavir/ritonavir and hydroxychloroquine (14).

Acute kidney injury (AKI) was observed in both patients during hospitalization. The risk of AKI increases with the concurrent use of CNIs as the basis of immunosuppression in kidney transplant recipients, especially those with high serum levels resulting from interaction with protease inhibitors (4). However, AKI is a common complication of COVID-19 (17) in patients who use CNIs. According to the findings of a recent study, lopinavir/ritonavir treatment had no benefit over standard care and even had more adverse effects (18).

We recommend discontinuation of m-TOR inhibitors as soon as possible and the addition of pulsatile corticosteroids. Furthermore, in the case of co-administration of CNIs or m-TOR inhibitors with strong CYP3A inhibitors, such as antivirals, close monitoring of blood concentrations, clinical status, and signs of drug toxicity are recommended. For additional reference throughout the COVID-19 pandemic, a summary of drug interactions with COVID-19 therapies is presented by the University of Liverpool (http://www.covid19-druginteractions.org/).

This study highlighted the importance of DDIs in kidney transplant patients with COVID-19. It is difficult to generalize the results to the whole transplant population; nevertheless, the findings of this and other reports can be used as guides for the management of such patients. In conclusion, a decrease in immunosuppressant dose should be measured in critically ill patients. Furthermore, special consideration is needed for the pharmaceutical interactions between antivirals, and immunesuppressants. In addition, frequent monitoring of CNI and m-TOR inhibitors levels during hospitalization is recommended to help determine ideal treatment and prevent serious clinical toxicity.

\section{References}

1. Michaels MG, La Hoz RM, Danziger-Isakov L, Blumberg EA, Kumar D, Green M, et al. Coronavirus disease 2019: implications of emerging infections for transplantation. $\mathrm{Am} \mathrm{J}$ Transplant. 2020;20(7):1768-72. doi: 10.1111/ajt.15832. [PubMed: 32090448].

2. Zhu L, Xu X, Ma K, Yang J, Guan H, Chen S, et al. Successful recovery of COVID-19 pneumonia in a renal transplant recipient with long-term immunosuppression. American Journal of Transplantation. 2020.

3. D'Antiga L. Coronaviruses and immunosuppressed patients: the facts during the third epidemic. Liver Transplantation. 2020 .

4. Naesens M, Kuypers DRJ, Sarwal M. Calcineurin inhibitor nephrotoxicity. Clin J Am Soc Nephrol. 2009;4(2):481-508. doi: 10.2215/cjn.04800908. [PubMed: 19218475].

5. Cossart AR, Cottrell WN, Campbell SB, Isbel NM, Staatz CE. Characterizing the pharmacokinetics and pharmacodynamics of immunosuppressant medicines and patient outcomes in elderly renal transplant patients. Transl Androl Urol. 2019; 8(Suppl 2):S198-213. doi: 10.21037/tau.2018.10.16. [PubMed: 
31236338]

6. Ju CR, Lian QY, Zhang JH, Qiu T, Cai ZT, Jiang WY, et al. Recommended prophylactic and management strategies for severe acute respiratory syndrome coronavirus 2 infection in transplant recipients. Chronic Dis Transl Med. 2020;6(2):87-97. doi: 10.1016/j.cdtm.2020.02.003. [PubMed: 32363045].

7. Dashti-Khavidaki S, Mohammadi K, Khalili H, Abdollahi A. Pharmacotherapeutic considerations in solid organ transplant patients with COVID-19. Expert Opinion on Pharmacotherapy. 2020;21(15):1813-9.

8. Meziyerh S, Zwart TC, van Etten RW, Janson JA, van Gelder T, Alwayn IP, et al. Severe COVID-19 in a renal transplant recipient: A focus on pharmacokinetics. Am J Transplant. 2020;20(7):1896-901. doi: 10.1111/ajt.15943. [PubMed: 32337790].

9. Bartiromo $\mathrm{M}$, Borchi B, Botta $\mathrm{A}$, Bagalà $\mathrm{A}$, Lugli G, Tilli $\mathrm{M}$, et al. Threatening drug-drug interaction in a kidney transplant patient with Coronavirus Disease 2019 (COVID-19). Transpl Infect Dis. 2020;22(4):e13286. doi: 10.1111/tid.13286. [PubMed: 32279418].

10. Russell CD, Millar JE, Baillie JK. Clinical evidence does not support corticosteroid treatment for 2019-nCoV lung injury. Lancet. 2020;395(10223):473-5. doi: 10.1016/S01406736(20)30317-2. [PubMed: 32043983].

11. Clarke A, Stein C, Townsend M. Drug-drug interactions with HIV antiretroviral therapy. US Pharm. 2008;33(4): HS-3-21.

12. Ventura-Aguiar P, Campistol JM, Diekmann F. Safety of mTOR inhibitors in adult solid organ transplantation. Expert Opin Drug Saf. 2016;15(3):303-19. doi: 10.1517/14740338.2016.1132698. [PubMed: 26667069].
13. Jain AB, Venkataramanan R, Eghtesad B, Marcos A, Ragni M, Shapiro $R$, et al. Effect of coadministered lopinavir and ritonavir (Kaletra) on tacrolimus blood concentration in liver transplantation patients. Liver Transpl. 2003;9(9):954-60. doi: 10.1053/jlts.2003.50171. [PubMed: 12942457].

14. van Maarseveen EM, Rogers CC, Trofe-Clark J, Van Zuilen AD, Mudrikova T. Drug-drug interactions between antiretroviral and immunosuppressive agents in HIV-infected patients after solid organ transplantation: a review. AIDS Patient Care STDS. 2012;26(10):568-81. doi: 10.1089/apc.2012.0169. [PubMed: 23025916].

15. Vogel M, Voigt E, Michaelis HC, Sudhop T, Wolff M, Türler A, et al. Management of drug-to-drug interactions between cyclosporine $\mathrm{A}$ and the protease-inhibitor lopinavir/ritonavir in liver-transplanted HIV-infected patients. Liver Transpl. 2004;10(7):939-44. doi: 10.1002/lt.20165. [PubMed: 15237382].

16. Finielz P, Gendoo Z, Chuet C, Guiserix J. Interaction between cyclosporin and chloroquine. Nephron. 1993;65(2):333. doi: 10.1159/000187506. [PubMed: 8247209].

17. Rodriguez-Morales AJ, Cardona-Ospina JA, Gutiérrez-Ocampo E, Villamizar-Peña R, Holguin-Rivera Y, Escalera-Antezana JP, et al. Clinical, laboratory and imaging features of COVID-19: a systematic review and meta-analysis. Travel Med Infect Dis. 2020;34:101623. doi: 10.1016/j.tmaid.2020.101623. [PubMed: 32179124].

18. Cao B, Wang Y, Wen D, Liu W, Wang J, Fan G, et al. A trial of lopinavir-ritonavir in adults hospitalized with severe Covid-19. $N$ Engl J Med. 2020;382(19):1787-99. doi: 10.1056/NEJMoa2001282. [PubMed: 32187464]. 